\title{
Die „Arbeitsgruppe für Ethik in der Psychiatrie“
}

\author{
Hartmann Hinterhuber
}

Online publiziert: 19. Dezember 2012

(C) Springer-Verlag Wien 2012

Die „Arbeitsgruppe für Ethik in der Psychiatrie" freut sich über die Initiative des Präsidenten, Herrn Prof. Dr. Christian Haring, das Tätigkeitsprofil der einzelnen Arbeitsgruppen zu erfassen und deren Wünsche entgegenzunehmen. Über Frau ÖÄ Dr. Regina Prunnlechner hat er ein diesbezügliches Rundschreiben an alle Verantwortlichen gerichtet.

Unsere Arbeitsgruppe hat sich die letzten Jahre erfolgreich bemüht, Ethik-Symposien in Zusammenarbeit mit der psychiatriehistorischen Arbeitsgruppe in Abstimmung mit den jährlichen Generalthemen der Jahreshauptversammlung der ÖGPP zu organisieren. Darüber hinaus bemühten wir uns, Ausbildungsärzte und Fachärzte für ethische Fragestellungen in der Psychiatrie durch Vorträge und Publikationen zu sensibilisieren (siehe beispielsweise: $\mathrm{H}$. Hinterhuber: Ethik in der Psychiatrie. In: Psychiatrie, Psychosomatik, Psychotherapie. Möller H.-J., Laux G., Kapfhammer H.-P. (Hrsg.) SpringerVerlag, 4. Auflage, S. 51-77, 2011).
Auch wurde ein ethischer Kodex für Psychiater im Auftrag der Österreichischen Gesellschaft für Psychiatrie und Psychotherapie erstellt (H. Hinterhuber et al.: Verhaltenscodex für Psychiater, Neuropsychiatrie 23: 4, 263-266, 2009). Ein weiterer Schwerpunkt war die Teilnahme an und Mitgestaltung von Veranstaltungen zu ethischen Fragestellungen innerhalb und außerhalb unserer Fachgesellschaft.

Unsere Arbeitsgruppe strebt für die kommenden 2 Jahre folgende Ziele an:

- Intensivierung der bisherigen Agenden.

- Implementierung von fachspezifischen Arbeitskreisen, die sich mit aktuellen ethischen Fragestellungen beispielsweise in der Kinder- und Jugendpsychiatrie, in der Gerontopsychiatrie und in der psychosozialen Versorgung beschäftigen.

- Die Forderung nach Inklusion von Menschen mit psychischen Stö- rungen zwingt zu verstärkter ethischer Reflexion.

- Verjüngung und Erweiterung der Mitgliederschaft der Arbeitsgruppe „Ethik in der Psychiatrie“.

Die angestrebten Ziele können nur dann effektiv angegangen werden, wenn in vermehrtem Umfang Kolleginnen und Kollegen motiviert werden können, sich intensiv mit den ethischen Fragen innerhalb unseres Faches zu beschäftigen. Alle Interessierten sind herzlich eingeladen, sich zu melden.

Wichtig erscheint ganz besonders, die ethischen Fragestellungen in der Psychiatrie nicht nur Medizinethikern, Bioethikern oder philosophischen Ethikern zu überlassen. Innensicht und Außensicht müssen sich ergänzen, wobei aber gerade der Innensicht eine ganz besondere Bedeutung zukommt. Psychiater dürfen sich vor ethischen Fragestellungen nicht drücken und die Beantwortung wesentlicher Fragen einzig den Berufsethikern überlassen.

em. Univ.-Prof. Dr. H. Hinterhuber $(\bowtie)$

Abteilung für Psychiatrie und Psychotherapie,

Medizinische Universität Innsbruck, Anichstraße 35,

6020 Innsbruck, Österreich

E-Mail: hartmann.hinterhuber@i-med.ac.at 